\title{
Association of IL-8 -251T>A (rs4073) polymorphism with susceptibility to gastric cancer: a systematic review and meta-analysis based on 33 case-control studies
}

\author{
Mansour MOGHIMI', Seyed Alireza DASTGHEIB ${ }^{2}$, Naeimeh HEIRANIZADEH ${ }^{3}$, Mohammad ZARE ${ }^{3}$, \\ Elnaz SHEIKHPOUR ${ }^{4}$ and Hossein NEAMATZADEH ${ }^{5,6}$
}

Received: 5 September 2019 Accepted: 4 November 2019

\begin{abstract}
Background - The role of -251A $>$ T polymorphism in the anti-inflammatory cytokine interleukin- 8 (IL-8) gene in gastric cancer was intensively evaluated, but the results of these studies were inconsistent. Objective - Therefore, we performed a meta-analysis to provide a comprehensive data on the association of IL-8 -251T >A polymorphism with gastric cancer. Methods - All eligible studies were identified in PubMed, Web of Science, EMBASE, Wanfang and CNKI databases before September 01, 2019. The pooled odds ratios (ORs) with $95 \%$ confidence intervals (CIs) were derived from a fixed effect or random effect model. Results - A total of 33 case-control studies with 6,192 cases and 9,567 controls were selected. Overall, pooled data showed that IL-8 -251T > A polymorphism was significantly associated with an increased risk of gastric cancer under all five genetic models, i.e., allele (A vs T: OR=1.189, 95\% CI 1.027-1.378, $P=0.021$ ), homozygote(AA vs TT: OR=1.307,95\% CI 1.111-1.536, $P=0.001$ ), heterozygote (AT vs TT: OR $=1.188,95 \% \mathrm{CI} 1.061-1.330$, $P=0.003$ ), dominant (AA+AT vs TT: $\mathrm{OR}=1.337,95 \% \mathrm{CI} 1.115-1.602, P=0.002$ ) and recessive (AA vs AT+TT: OR=1.241, 95\% CI 1.045-1.474, $P=0.014$ ). The stratified analysis by ethnicity revealed an increased risk of gastric cancer in Asians and mixed populations, but not in Caucasians. Moreover, stratified by country found a significant association in Chinese, Korean and Brazilian, but not among Japanese. Conclusion - This meta-analysis suggests that the IL-8 -251T >A polymorphism is associated with an increased risk of gastric cancer, especially by ethnicity (Asian and mixed populations) and country (Chinese, Korean and Brazilian).
\end{abstract}

HEADINGS - Stomach neoplasms. Interleukin 8. Genetic polymorphism. Meta-analysis.

\section{INTRODUCTION}

Gastric cancer is the 4th most common malignancy and the second leading cause of cancer-related death worldwide ${ }^{(1,2)}$, accounting for more than one million new patients and an estimated 783,000 deaths in $2018^{(3)}$. There is considerable variation in gastric cancer incidence rates according to age, gender, socioeconomical factors and geographical location ${ }^{(4)}$. Several factors have been suggested as risk factors for gastric cancer, which by establishing complex interactions may ultimately lead to development of this disease. The exact etiology of gastric cancer is multifactorial and both host genetic variants and environmental factors, including inflammation, Helicobacter pylori (H. pylori) infection, cigarette smoking, alcohol consumption, dietary and nutritional aspects have been shown to play a role in the development of this disease $\mathrm{e}^{(5,6)}$.

Interleukin-8 (IL-8, also known as CXCL8) is a member of the alpha (C-X-C) subfamily of small basic heparin-binding chemokines ${ }^{(7)}$. These group of proteins are proinflammatory and primarily mediate the activation and migration of neutrophils into tissue from peripheral blood ${ }^{(8)}$. IL-8 was originally discovered and purified as a neutrophil chemotactic and activating factor and secreted by several hematopoietic cells, fibroblasts, hepatocytes, and various cell lines and interacts with two specific seven-transmembrane span, G-protein-coupled receptors, CXCR1 and CXCR2 $2^{(9,10)}$. Moreover, IL- 8 is produced by several types of tumor cells and has been shown to be involved in angiogenesis and neovascularizationdependent tumor growth ${ }^{(11)}$. Hence, IL-8 gene variants may be the important determinants of development of different cancers, especially gastric cancer. IL-8 is associated with both the immune response and the inflammatory process against $H$. pylori ${ }^{(12)}$. An increased IL-8 expression has been detected in patients with H. pylori infection and gastric disorders ${ }^{(12,13)}$.

The human IL- 8 gene is mapped to 4 q12-q21 by somatic cell hybridization and in situ hybridization, spanning $5.2 \mathrm{~kb}$ in length and contains 10 exons $^{(14)}$. The 3' untranslated region (UTR) of IL-8 contains a A/U-rich element that makes it extremely unstable under certain conditions. The -251T >A (rs4073) polymorphism in the IL-8 promoter is one of the most extensively studied genetic variant in several inflammatory conditions ${ }^{(15)}$. Although several studies have been previously performed to evaluate whether the IL-8 -251T>A (rs4073) polymorphism increases the risk of gastric cancer, the results from these studies are inconsistent and controversial. Therefore, this study aimed to perform a meta-analysis including the update data to evaluate the association of IL-8 $-251 \mathrm{~T}>\mathrm{A}$ (rs4073) polymorphism and gastric cancer.

Shahid Sadoughi University of Medical Sciences, Department of Pathology, Yazd, Iran. ${ }^{2}$ Shiraz university of Medical Sciences, Department of Medical Genetics, Shiraz, Iran. ${ }^{3}$ Shahid Sadoughi University of Medical Sciences, Department of Surgery, Yazd, Iran. ${ }^{4}$ Shahid Sadoughi University of Medical Science, Hematology and Oncology Research Center, Yazd, Iran. ${ }^{5}$ Shahid Sadoughi University of Medical Sciences, Department of Medical Genetics, Yazd, Iran. ${ }^{6}$ Shahid Sadoughi University of Medical Sciences, Mother and Newborn Health Research Center, Yazd, Iran.

Corresponding author: Dr. Seyed Alireza Dastgheib. E-mail: dastgheibsa@gmail.com 


\section{METHODS}

\section{Search strategy}

We have performed this meta-analysis in adherence with the PRISMA (Preferred Reporting Items for Systematic Reviews and Meta-Analyses) guidelines. A comprehensive literature search was conducted in PubMed, EMBASE, Cochrane Library database, SID, CBM, WanFang Chinese Biomedical Database (CBD), China National Knowledge Infrastructure (CNKI) and VIP database to collect all the eligible studies evaluating the association of IL-8 -251T >A (rs4073) polymorphism and gastric cancer until to September 01, 2019. The following terms, keywords and their combinations were used: ("gastric" OR "stomach") AND ("cancer" OR "malignancy" OR "tumor" OR "carcinoma" or "neoplasm" OR "adenocarcinoma") AND ("interleukin-8" OR "IL-8" OR "C-X-C motif chemokine ligand 8" OR "CXCL8" OR "neutrophil chemotactic factor") AND ("polymorphism" OR "SNP" OR "variant" OR "genotype" OR "mutation" OR "allele"). The whole search process was carried out in English, Chinese and Farsi. Ethical approval was not necessary since this study was based on previous publications.

\section{Inclusion and exclusion criteria}

The studies were considered eligible if the following criteria are met: 1) studies with case-control and cohort design; 2) studies were performed on human beings; 3 ) studies evaluated the association between the IL-8 -251T > A polymorphism and gastric cancer; and 4) provide adequate data to calculate odds ratios (ORs) and $95 \%$ confidence intervals (CIs). Studies were excluded if one of the following criteria was fulfilled: 1) studies that had no control group; 2) abstracts, review, letters, comments, conference presentations, case reports or case series; 3) studies without detailed raw data regarding IL-8 -251T>A polymorphism; 4) family-based, sibling, twins and linkage studies. For duplicate reports, only the study with the largest sample size was included.

\section{Data extraction}

All the available data were extracted from each study by two of the authors independently and carefully according to the criteria and any disagreement was resolved by discussion with third author. For each included study, the following information was collected: first author's name, year of publication, ethnicity (Asian, Caucasian, African and mixed populations), country of origin, source of the controls (hospital based or population based), genotyping methods, numbers of cases and controls, frequencies of genotypes in cases and controls, minor allele frequency (MAF) in controls, and Hardy-Weinberg equilibrium (HWE) in controls. The "mixed" group means mixed or unknown populations. When studies included sample of more than one ethnicity or population, the data was extracted separately according to ethnicities. If necessary data were not reported in the primary manuscripts, we contacted the corresponding authors by email to request the missing data.

\section{Methodological quality assessment}

Quality assessments for eligible studies were conducted by two investigators independently using the Newcastle-Ottawa Scale (NOS). In this methodological quality assessment scale, nine items, each with a score value between one and nine, are included. The NOS has a score range of zero to nine, and studies with a NOS score of $\geq 6$ stars is generally considered of high-quality.

\section{Statistical analysis}

The strength of association between IL-8 -251T>A poly- morphism and gastric cancer was assessed by odds ratios (ORs) with $95 \%$ confidence intervals $(95 \%$ CIs). The significance of the pooled effect size was determined by Z-test, in which $P<0.05$ was considered statistically significant. The association was evaluated in five genetic models, i.e., allele (A vs T), homozygote (AA vs TT), heterozygote (AT vs TT), dominant (AA+AT vs TT), and the recessive (AA vs AT+TT). Between-study heterogeneity was evaluated by the Cochran Q-test, in which $P \leq 0.10$ indicated significant heterogeneity was found. In addition, the $\mathrm{I}^{2}$ statistic we applied to qualify between-study heterogeneity (range of 0 to $100 \%$ : $\mathrm{I}^{2}=0-25 \%$, no heterogeneity; $\mathrm{I}^{2}=25 \%-50 \%$, moderate heterogeneity; $\mathrm{I}^{2}=50 \%-75 \%$, large heterogeneity; $\mathrm{I}^{2}=75 \%-100 \%$, extreme heterogeneity). The random effects model shows more flexibility with respect to variable effect size in different studies and study populations. Thus, we have applied a random-effects model, using the DerSimonian and Laird method to calculate the pooled OR when heterogeneity was found; otherwise, affixed effect model was applied to use the Mantel-Haenszel method in absence of heterogeneity. A Hardy-Weinberg equilibrium (HWE) test in controls was tested using chi-square test $(P$-values $<0.05)$. Subgroup analyses were conducted by stratification of ethnicity to identifying potential source of heterogeneity. Sensitivity analyses were performed to assess influence of each single study on pooled ORs and the stability of the meta-analysis results by sequential remove of individual studies. In addition, sensitivity analysis by excluding those studies HWE violating was performed to examine the stability of the results. Funnel plots and Egger's linear regression test were used to estimate evidence for potential publication bias. All of the statistical calculations were performed using Comprehensive Meta-Analysis (CMA) software version 2.0 (Biostat, USA). Two-sided $P$-values $<0.05$ were considered statistically significant.

\section{RESULTS}

\section{Characteristics of selected studies}

FIGURE 1 shows the flowchart of literature search and selection process. The initial literature searches retrieved 158 potentially relevant studies. After reading titles and abstracts, 67 irrelevant and duplicate articles were excluded. Another 32 articles were subse-

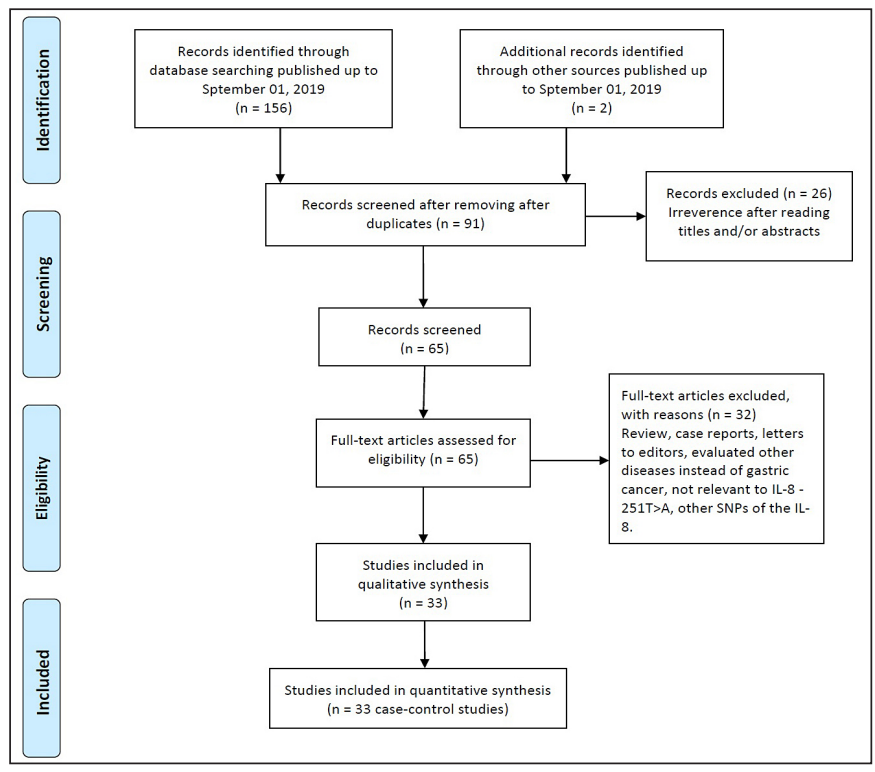

FIGURE 1. Flowchart of literature search and selection process. 
quently excluded because not reporting useful data for meta-analysis, review, case only study, and not being case-control studies. Finally, a total of 33 eligible studies with 6,192 gastric cancer cases and 9,567 controls were included in the meta-analysis ${ }^{(16-39)}$. Characteristics of included studies are shown in TABLE 1. All eligible studies were published in English and Chinese. The NOS score of included studies ranged from 7 ( 19 studies) to 8 (14 studies), which suggested that all included studies were of relatively high quality. Among them, 21 studies were based on Asians (Taiwan, Japan, Korea, Iran, and India), seven based on Caucasians (USA, Finland, France, Portugal, Hungary, Romanian), and five were based on mixed populations
(Mexico and Brazil). The allele, genotype and minor allele frequency (MAF) distributions in the cases and controls are shown in TABLE 1. Moreover, the distribution of genotypes in the controls was in agreement with Hardy-Weinberg equilibrium (HWE) for all selected studies, except for one study (TABLE 1).

\section{Overall and subgroup analyses}

The summary of the meta-analysis of the association of between IL-8 -251T >A polymorphism and gastric cancer are shown in TABLE 2. Overall, pooled ORs showed that there was a significant association between IL-8 -251T>A polymorphism and gastric can-

TABLE 1. Main characteristics of studies included in the meta-analysis.

\begin{tabular}{|c|c|c|c|c|c|c|c|c|c|c|c|c|c|c|c|c|c|}
\hline \multirow{3}{*}{ First author } & \multirow{3}{*}{$\begin{array}{c}\text { Country } \\
\text { (Ethnicity) }\end{array}$} & \multirow{3}{*}{$\begin{array}{l}\text { Genotyping } \\
\text { Method }\end{array}$} & \multirow{3}{*}{ SOC } & \multirow{3}{*}{$\begin{array}{c}\text { Case/ } \\
\text { Control }\end{array}$} & \multicolumn{5}{|c|}{ Cases } & \multicolumn{5}{|c|}{ Controls } & \multirow{3}{*}{ MAFs } & \multirow{3}{*}{ HWE } & \multirow{3}{*}{ NOS } \\
\hline & & & & & \multicolumn{3}{|c|}{ Genotypes } & \multicolumn{2}{|c|}{ Allele } & \multicolumn{3}{|c|}{ Genotypes } & \multicolumn{2}{|c|}{ Allele } & & & \\
\hline & & & & & TT & $\mathrm{TA}$ & AA & $\mathrm{T}$ & $\mathrm{A}$ & TT & TA & AA & $\mathrm{T}$ & $A$ & & & \\
\hline \multirow{2}{*}{ Savage 2004} & USA & SBE & $\mathrm{PB}$ & $88 / 429$ & 26 & 39 & 23 & 91 & 85 & 147 & 207 & 75 & 501 & 357 & 0.416 & 0.884 & 7 \\
\hline & (Caucasian) & TaqMan & PB & $287 / 428$ & 76 & 140 & 71 & 282 & 292 & 117 & 205 & 106 & 439 & 417 & 0.487 & 0.391 & 7 \\
\hline Lee 2005 & $\begin{array}{l}\text { Taiwan } \\
\text { (Asian) }\end{array}$ & PCR-RFLP & HB & $364 / 291$ & 156 & 164 & 44 & 609 & 331 & 108 & 127 & 56 & 354 & 262 & 0.411 & 0.093 & 8 \\
\hline $\begin{array}{l}\text { Taguchi } \\
2005\end{array}$ & Japan (Asian) & PCR-RFLP & $\mathrm{HB}$ & $396 / 215$ & 161 & 191 & 44 & 513 & 279 & 125 & 105 & 22 & 355 & 149 & 0.296 & 0.994 & 7 \\
\hline Lu 2005 & China (Asian) & PCR-DHPLC & PB & $250 / 300$ & 94 & 102 & 54 & 290 & 210 & 119 & 144 & 37 & 382 & 218 & 0.363 & 0.515 & 8 \\
\hline Zeng 2005 & China (Asian) & PCR-RDB & PB & $206 / 504$ & 37 & 110 & 59 & 184 & 228 & 43 & 114 & 39 & 200 & 192 & 0.490 & 0.021 & 8 \\
\hline Leung 2006 & China (Asian) & TaqMan & NS & $123 / 179$ & 44 & 56 & 23 & 144 & 102 & 51 & 92 & 36 & 194 & 164 & 0.467 & 0.835 & 7 \\
\hline $\begin{array}{l}\text { Ohyauchi } \\
2005\end{array}$ & Japan (Asian) & DS & $\mathrm{HB}$ & $212 / 244$ & 93 & 106 & 13 & 292 & 132 & 149 & 84 & 11 & 534 & 158 & 0.217 & 0.847 & 7 \\
\hline $\begin{array}{l}\text { Shirai } 2005 \\
\text { Kamali- }\end{array}$ & Japan (Asian) & PCR-RFLP & $\mathrm{HB}$ & $181 / 468$ & 83 & 78 & 20 & 244 & 118 & 211 & 208 & 49 & 630 & 306 & 0.327 & 0.830 & 7 \\
\hline $\begin{array}{l}\text { Sarvestani } \\
2006\end{array}$ & Iran (Asian) & ASO-PCR & $\mathrm{HB}$ & $19 / 153$ & 4 & 6 & 9 & 14 & 24 & 57 & 74 & 22 & 188 & 118 & 0.386 & 0.797 & 7 \\
\hline $\begin{array}{l}\text { Kamangar } \\
2006\end{array}$ & $\begin{array}{c}\text { Finland } \\
\text { (Caucasian) }\end{array}$ & TaqMan & PB & $112 / 207$ & 42 & 56 & 14 & 140 & 84 & 72 & 111 & 24 & 255 & 159 & 0.384 & 0.054 & 8 \\
\hline $\begin{array}{l}\text { Garza- } \\
\text { Gonzalez } \\
2007\end{array}$ & Mexico (Mixed) & ARMS-PCR & HB & $78 / 189$ & 15 & 47 & 16 & 77 & 79 & 69 & 87 & 33 & 225 & 153 & 0.405 & 0.538 & 7 \\
\hline Crusius 2008 & $\begin{array}{c}\text { France } \\
\text { (Caucasian) }\end{array}$ & Real-Time & PB & $236 / 1139$ & 75 & 113 & 48 & 263 & 209 & 315 & 574 & 250 & 1204 & 1074 & 0.471 & 0.705 & 8 \\
\hline Canedo 2008 & $\begin{array}{c}\text { Portugal } \\
\text { (Caucasian) }\end{array}$ & TaqMan & PB & $333 / 693$ & 111 & 169 & 53 & 391 & 275 & 203 & 353 & 137 & 759 & 627 & 0.452 & 0.459 & 7 \\
\hline Szoke 2008 & $\begin{array}{l}\text { Hungary } \\
\text { (Caucasian) }\end{array}$ & ARMS-PCR & NS & $35 / 168$ & 11 & 15 & 9 & 37 & 33 & 38 & 93 & 37 & 169 & 167 & 0.497 & 0.164 & 7 \\
\hline Kang 2009 & Korea (Asian) & PCR-RFLP & PB & $334 / 322$ & 126 & 159 & 49 & 411 & 257 & 147 & 148 & 27 & 442 & 202 & .314 & 0.225 & 8 \\
\hline Ko 2009 & Korea (Asian) & Snapshot & PB & $81 / 308$ & 34 & 35 & 12 & 103 & 59 & 135 & 146 & 27 & 416 & 200 & .325 & 0.155 & 8 \\
\hline Ye 2009 & Korea (Asian) & PCR-RFLP & $\mathrm{HB}$ & $53 / 206$ & 54 & 82 & 17 & 190 & 116 & 97 & 86 & 23 & 280 & 132 & 0.320 & .552 & 7 \\
\hline Song 2009 & China (Asian) & PCR-RFLP & $\mathrm{HB}$ & $25 / 140$ & 33 & 72 & 20 & 138 & 112 & 47 & 70 & 23 & 164 & 116 & 0.414 & 0.720 & 8 \\
\hline Liu 2009 & China (Asian) & TaqMan & HB & $138 / 137$ & 26 & 89 & 23 & 141 & 135 & 50 & 72 & 15 & 172 & 102 & 0.372 & 0.145 & 7 \\
\hline Li 2010 & China (Asian) & PCR-DHPLC & NS & $101 / 137$ & 25 & 65 & 11 & 115 & 87 & 59 & 64 & 14 & 184 & 92 & 0.336 & 0.579 & 7 \\
\hline Bo 2010 & China (Asian) & PCR-RFLP & $\mathrm{HB}$ & 208/190 & 64 & 108 & 36 & 236 & 180 & 68 & 96 & 26 & 232 & 148 & 0.386 & 0.389 & 8 \\
\hline Zhang 2010 & China (Asian) & PCR-RFLP & PB & $519 / 504$ & 130 & 261 & 128 & 521 & 517 & 160 & 251 & 93 & 571 & 437 & 0.434 & 0.754 & 8 \\
\hline Vinagre 2011 & Brazil (Mixed) & PCR-RFLP & $\mathrm{HB}$ & $102 / 103$ & 21 & 56 & 25 & 98 & 106 & 42 & 42 & 19 & 126 & 80 & 0.387 & 0.122 & 8 \\
\hline Felipe 2012 & Brazil (Mixed) & PCR-RFLP & $\mathrm{PB}$ & $104 / 196$ & 31 & 58 & 15 & 120 & 88 & 59 & 85 & 52 & 203 & 189 & 0.482 & 0.065 & 7 \\
\hline Burada 2012 & $\begin{array}{c}\text { Romania } \\
\text { (Caucasian) }\end{array}$ & Real-Time & $\mathrm{HB}$ & $105 / 242$ & 31 & 54 & 20 & 116 & 94 & 82 & 112 & 48 & 276 & 208 & 0.430 & 0.385 & 7 \\
\hline $\operatorname{ran} 2014$ & China (Asian) & SBE & ПD & 201 & 92 & 168 & 48 & 352 & 264 & 101 & 148 & 59 & 350 & 266 & 0.432 & 0.715 & 7 \\
\hline Qadri 2014 & India (Asian) & PCR-CTPP & $\mathrm{PB}$ & $130 / 200$ & 50 & 68 & 12 & 168 & 92 & 94 & 94 & 12 & 282 & 118 & 0.295 & 0.066 & 8 \\
\hline Kumar 2015 & India (Asian) & ASO-PCR & $\mathrm{HB}$ & $200 / 250$ & 67 & 86 & 47 & 220 & 180 & 93 & 122 & 35 & 308 & 192 & 0.384 & 0.618 & 8 \\
\hline $\begin{array}{l}\text { de Oliveira } \\
2015\end{array}$ & Brazil (Mixed) & PCR-RFLP & $\mathrm{HB}$ & $240 / 207$ & 61 & 134 & 45 & 222 & 192 & 62 & 98 & 47 & 256 & 224 & 0.464 & 0.488 & 7 \\
\hline Wang 2016 & China (Asian) & MassARRAY & HB & $132 / 296$ & 47 & 59 & 26 & 153 & 111 & 102 & 144 & 50 & 348 & 244 & 0.412 & 0.945 & 7 \\
\hline Ramis 2017 & Brazil (Mixed) & PCR-RFLP & $\mathrm{PB}$ & $9 / 38$ & 4 & 1 & 4 & 9 & 9 & 11 & 20 & 7 & 42 & 34 & 0.447 & 0.691 & 7 \\
\hline Chang 2017 & Korea (Asian) & PCR-RFLP & $\mathrm{HB}$ & $283 / 176$ & 81 & 168 & 34 & 330 & 236 & 70 & 89 & 17 & 229 & 123 & 0.349 & 0.136 & 8 \\
\hline
\end{tabular}

SBE: allele-specific single base extension; PCR: polymerase chain reaction; RFLP: polymerase chain reaction restriction fragment length polymorphism; DHPLC: denaturing high performance liquid chromatography; DS: direct sequencing; ASO: Allele-specific oligonucleotide; ARMS: amplification refractory mutation system; SOC: source of controls; HB: hospital-based; PB: population-based; NS: not stated; MAFs: minor allele frequencies; HWE: Hardy-Weinberg equilibrium; NOS: Newcastle-Ottawa Scale. 
TABLE 2. Summary of meta-analysis for association between IL-8 -251T > A polymorphism and gastric cancer risk.

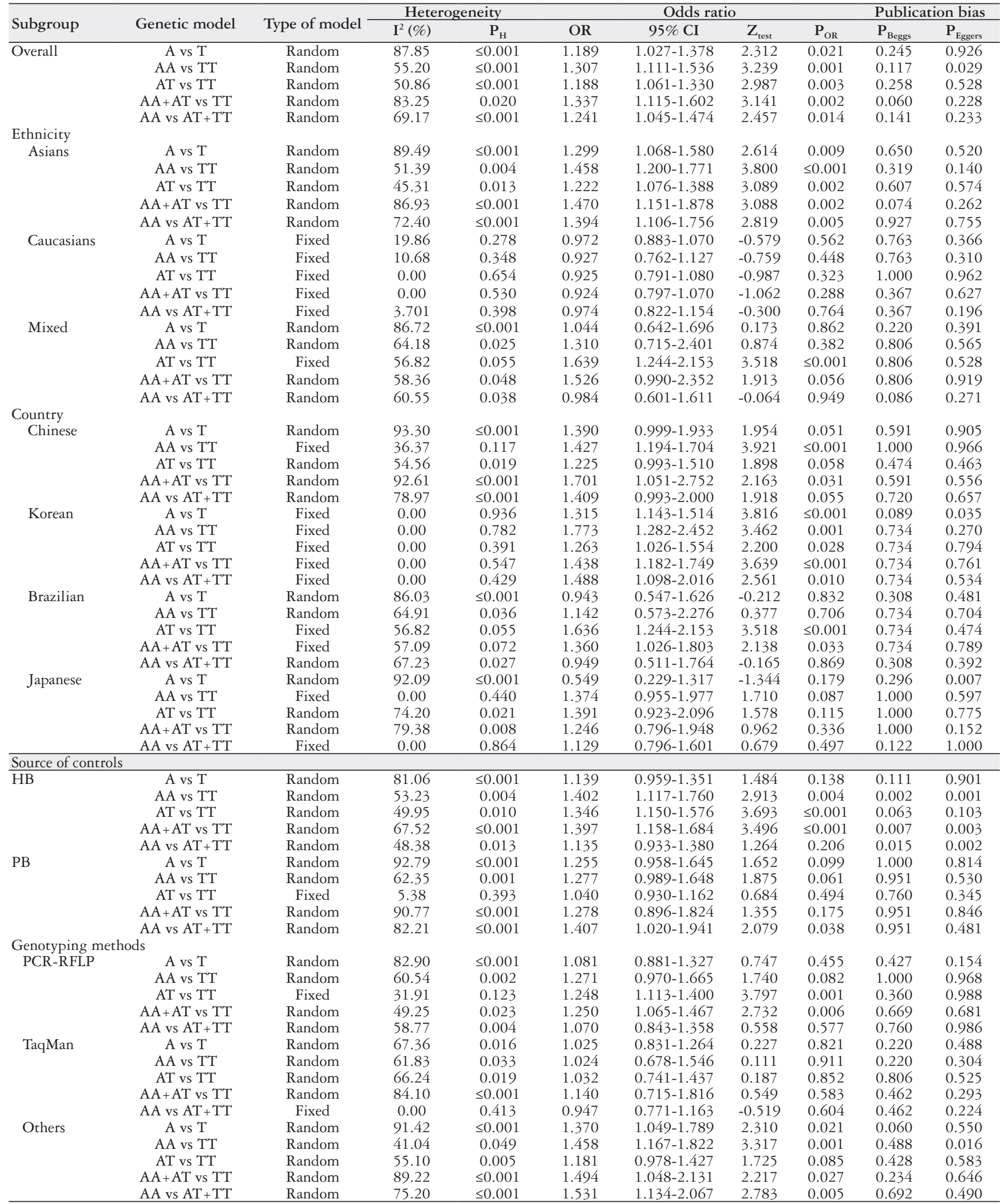


cer under all five genetic models, i.e., allele (A vs T: $O R=1.189,95 \%$ CI 1.027-1.378, $P=0.021$, FIGURE 2A), homozygote (AA vs TT: $\mathrm{OR}=1.307,95 \% \mathrm{CI} 1.111-1.536, P=0.001$ ), heterozygote (AT vs TT: $\mathrm{OR}=1.188,95 \% \mathrm{CI} 1.061-1.330, P=0.003)$, dominant $(\mathrm{AA}+\mathrm{AT}$ vs TT: $\mathrm{OR}=1.337,95 \% \mathrm{CI} 1.115-1.602, P=0.002)$ and recessive (AA vs AT+TT: $\mathrm{OR}=1.241,95 \%$ CI $1.045-1.474, P=0.014)$.

Moreover, we have performed subgroup analysis by ethnicity, country (China, Korea, Japan and Brazil), source of controls, and genotyping methods. Stratified analysis by ethnicity revealed that there was a significant association between IL-8 -251T >A polymorphism and gastric cancer in Asian (A vs T: OR=1.299, 95\% CI $1.068-1.580, P=0.009$; AA vs TT: OR $=1.458,95 \%$ CI $1.200-1.771, P \leq 0.001$, FIGURE $2 \mathrm{~B}$; AT vs TT: $\mathrm{OR}=1.222,95 \%$ CI $1.076-1.388, P=0.002$; AA+AT vs TT: $\mathrm{OR}=1.470,95 \% \mathrm{CI}$ $1.151-1.878, P=0.002$; and AA vs AT+TT: OR $=1.394,95 \% \mathrm{CI}$ $1.106-1.756, P=0.005$ ), mixed populations (AT vs TT: $\mathrm{OR}=1.639$, $95 \%$ CI 1.244-2.153, $P \leq 0.001)$, but not in Caucasians. Moreover, subgroup analysis by country showed a significant association between IL-8 -251T $>$ A polymorphism and gastric cancer in Chinese (AA vs TT: $\mathrm{OR}=1.427,95 \%$ CI $1.194-1.704, P \leq 0.001$; and AA+AT vs TT: $\mathrm{OR}=1.701,95 \%$ CI $1.051-2.752, P=0.031$, FIGURE 2C), Korean (A vs T: OR=1.315, 95\% CI 1.143-1.514,
$P \leq 0.001 ;$ AA vs TT: $\mathrm{OR}=1.773,95 \%$ CI $1.282-2.452, P=0.001$; AT vs TT: $\mathrm{OR}=1.263,95 \%$ CI 1.026-1.554, $P=0.028$; AA+AT vs TT: $\mathrm{OR}=1.438,95 \%$ CI $1.182-1.749, P \leq 0.001$; and AA vs AT+TT: $\mathrm{OR}=1.488,95 \%$ CI 1.098-2.016, $P=0.010$ ) and in Brazilian (AT vs TT: $\mathrm{OR}=1.636,95 \%$ CI 1.244-2.153, $P \leq 0.001$, FIGURE 2D; and AA+AT vs TT: $\mathrm{OR}=1.360,95 \%$ CI $1.026-1.803, P=0.033$ ), but not in Japanese. When stratified by source of controls, the results showed a significant association between IL-8 -251T >A polymorphism and gastric cancer in hospital based studies (AA vs TT: $\mathrm{OR}=1.402,95 \%$ CI $1.117-1.760, P=0.004 ;$ AT vs TT: $\mathrm{OR}=1.346$, 95\% CI $1.150-1.576, P \leq 0.001$; and AA+AT vs TT: OR=1.397, 95\% CI 1.158-1.684, $P \leq 0.001)$ and population based studies (AA vs AT+TT: $\mathrm{OR}=1.407,95 \%$ CI $1.020-1.941, P=0.038)$. Subgroup analysis by genotyping methods revealed that there was a significant association between IL-6 $-176 \mathrm{G}>\mathrm{C}$ polymorphism and gastric cancer risk in PCR-RFLP group of studies (AT vs TT: $\mathrm{OR}=1.248$, 95\% CI 1.113-1.400, $P=0.001$; and AA+AT vs TT: $O R=1.250$, $95 \%$ CI 1.065-1.467, $P=0.006)$ and in group of studies used other genotyping methods (A vs T: OR $=1.370,95 \%$ CI 1.049-1.789, $P=0.021$; AA vs TT: $\mathrm{OR}=1.457,95 \%$ CI $1.167-1.822, P=0.001$; AA+AT vs TT: $\mathrm{OR}=1.494,95 \%$ CI $1.048-2.131, P=0.027$; and AA vs $\mathrm{AT}+\mathrm{TT}$ : $\mathrm{OR}=1.531,95 \% \mathrm{CI} 1.134-2.067, P=0.005)$.

\section{A Study name}

$\begin{array}{lrrrrr} & \begin{array}{c}\text { Odds } \\ \text { ratio }\end{array} & \begin{array}{c}\text { Lower } \\ \text { limit }\end{array} & \begin{array}{c}\text { Upper } \\ \text { limit }\end{array} & \text { Z-Value } & \text { p-Value } \\ \text { Savage 2004a } & 1.311 & 0.947 & 1.815 & 1.631 & 0.103 \\ \text { Savage 2004b } & 1.090 & 0.882 & 1.347 & 0.799 & 0.424 \\ \text { Lee 2005 } & 1.018 & 0.818 & 1.268 & 0.163 & 0.871 \\ \text { Taguchi 2005 } & 1.026 & 0.802 & 1.312 & 0.202 & 0.840 \\ \text { Lu 2005 } & 1.269 & 0.995 & 1.618 & 1.918 & 0.055 \\ \text { Zeng 2005 } & 5.266 & 4.102 & 6.761 & 13.031 & 0.000 \\ \text { Leung 2006 } & 0.838 & 0.604 & 1.163 & 1.057- & 0.291 \\ \text { Ohyauchi 2005 } & 0.944 & 0.714 & 1.249 & 0.403- & 0.687 \\ \text { Shirai 2006 } & 0.001 & 0.000 & 0.013 & 5.031- & 0.000 \\ \text { Kamali-Sarvestani } 2006 & 4.300 & 2.151 & 8.598 & 4.127 & 0.000 \\ \text { Kamangar 2006 } & 0.962 & 0.688 & 1.346 & 0.225- & 0.822 \\ \text { Garza-Gonzalez 2007 } & 1.509 & 1.037 & 2.195 & 2.149 & 0.032 \\ \text { Crusius 2008 } & 0.891 & 0.730 & 1.087 & 1.136- & 0.256 \\ \text { Canedo 2008 } & 0.851 & 0.706 & 1.026 & 1.686- & 0.092 \\ \text { Szoke 2008 } & 0.903 & 0.539 & 1.512 & 0.390- & 0.697 \\ \text { Kang 2009 } & 1.368 & 1.089 & 1.719 & 2.695 & 0.007 \\ \text { Ko 2009 } & 1.191 & 0.830 & 1.711 & 0.949 & 0.343 \\ \text { Ye 2009 } & 1.295 & 0.950 & 1.766 & 1.634 & 0.102 \\ \text { Song 2009 } & 1.147 & 0.813 & 1.619 & 0.782 & 0.434 \\ \text { Liu 2009 } & 1.615 & 1.149 & 2.269 & 2.760 & 0.006 \\ \text { Li 2010 } & 1.497 & 1.029 & 2.177 & 2.109 & 0.035 \\ \text { Bo 2010 } & 1.283 & 0.964 & 1.706 & 1.710 & 0.087 \\ \text { Zhang 2010 } & 1.297 & 1.089 & 1.543 & 2.924 & 0.003 \\ \text { Vinagre 2011 } & 1.704 & 1.151 & 2.522 & 2.661 & 0.008 \\ \text { Felipe 2012 } & 0.788 & 0.561 & 1.105 & 1.380- & 0.168 \\ \text { Burada 2012 } & 1.075 & 0.776 & 1.490 & 0.436 & 0.663 \\ \text { Pan 2014 } & 0.987 & 0.788 & 1.237 & 0.115- & 0.908 \\ \text { Qadri 2014 } & 1.309 & 0.938 & 1.826 & 1.584 & 0.113 \\ \text { Kumar 2015 } & 1.313 & 1.005 & 1.714 & 1.996 & 0.046 \\ \text { de Oliveira 2015 } & 0.565 & 0.433 & 0.738 & 4.202- & 0.000 \\ \text { Wang 2016 } & 1.035 & 0.771 & 1.389 & 0.227 & 0.820 \\ \text { Ramis 2017 } & 1.235 & 0.442 & 3.455 & 0.403 & 0.687 \\ \text { Chang 2017 } & 1.331 & 1.011 & 1.754 & 2.036 & 0.042 \\ & 1.189 & 1.027 & 1.378 & 2.312 & 0.021 \\ & & & & & \\ & & & & & \\ & & & & & \end{array}$

Odds ratio and $95 \% \mathrm{Cl}$

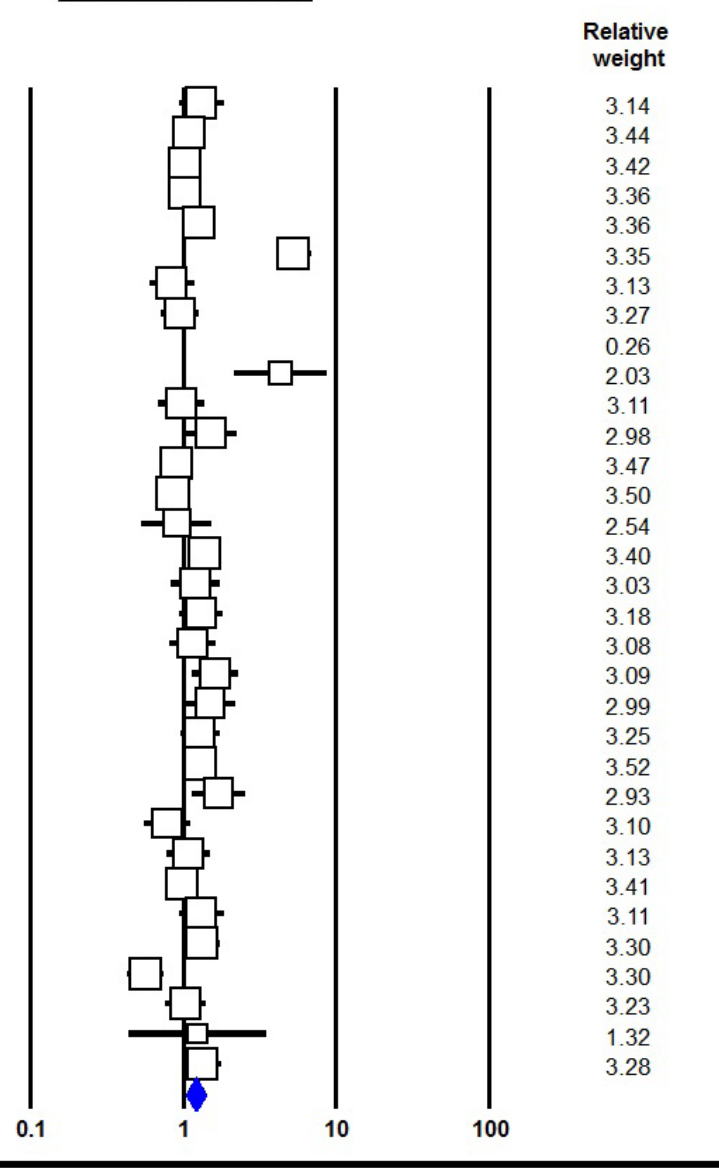

FIGURE 2. Forest plot for association of IL-8 -251T>A polymorphism and gastric cancer. A: in overall population (A vs T). 


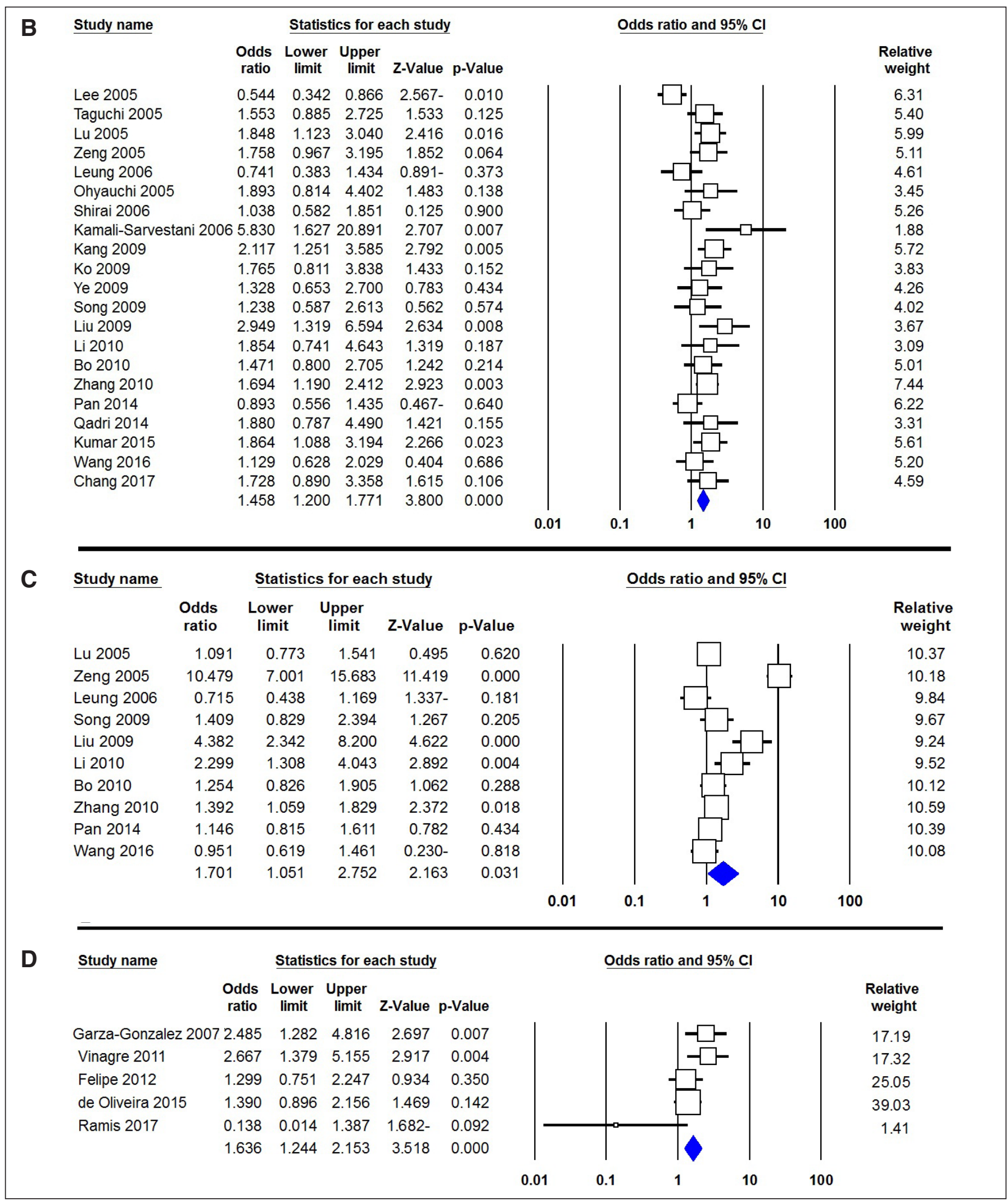

FIGURE 2. Forest plot for association of IL-8 $-251 \mathrm{~T}>\mathrm{A}$ polymorphism and gastric cancer. B: in Asians (AA vs TT). C: in Chinese (AA+AT vs TT). D: in Brazilian (AT vs TT). 


\section{Between-study heterogeneity}

There was statistically moderate to high heterogeneity among the studies under all five genetic models, i.e., allele $\left(\mathrm{I}^{2}=87.85\right.$, $\left.\mathrm{P}_{\mathrm{H}} \leq 0.001\right)$, homozygote $\left(\mathrm{I}^{2}=55.20, \mathrm{P}_{\mathrm{H}} \leq 0.001\right)$, heterozygote $\left(\mathrm{I}^{2}=50.86, \mathrm{P}_{\mathrm{H}} \leq 0.001\right)$, dominant $\left(\mathrm{I}^{2}=83.25, \mathrm{P}_{\mathrm{H}} \leq 0.001\right)$, and recessive $\left(\mathrm{I}^{2}=69.17, \mathrm{P}_{\mathrm{H}} \leq 0.001\right)$ in overall population. To explore the sources of heterogeneity, we performed subgroup analyses using ethnicity, country, source of controls and genotyping methods. The results suggested that ethnicity and country (population) may contribute to the heterogeneity in this meta-analysis.

\section{Sensitivity analysis}

To evaluate the sensitivity of the meta-analysis, we omitted each study at a time and checked for significant differences. There were no significant differences observed upon removal of any of the studies, indicating that our results are statistically reliable and stable. Deviation of HWE may reflect methodological problem such as genotyping errors, population stratification or selection bias. Moreover, we performed sensitivity analysis by excluding the HWE-violating study. When this study was excluded, the results were not changed in overall population and also by subgroup analyses, indicating that our meta-analysis was statistically robust and reliable.

\section{Publication bias}

Publication bias was assessed with Begg's funnel plots and Egger's test (TABLE 2). The results of Begg's funnel plots and Egger's regression test suggested evidence of publication bias in overall population under homozygote genetic model (AA vs TT: $\mathrm{P}_{\text {Beggs }}=0.117 ; \mathrm{P}_{\text {Eggers }}=0.029 ;$ FIGURE 3$)$, and by subgroup analysis in Korean under allele model (A vs T: $\mathrm{P}_{\text {Beggs }}=0.089$; $\mathrm{P}_{\text {Eggers }}=0.035$ ), Japanese under allele model (A vs T: $\mathrm{P}_{\text {Beggs }}=0.296$; $\left.\mathrm{P}_{\text {Eggers }}=0.007\right)$, hospital based studies under homozygote model (AA vs TT: $\left.\mathrm{P}_{\text {Begos }}=0.002 ; \mathrm{P}_{\text {Eggers }}=0.001\right)$, dominant $(\mathrm{AA}+\mathrm{AT}$ vs TT: $\left.P_{\text {Beggs }}=0.007 ; \mathrm{P}_{\text {Eggers }}=0.003\right)$ and recessive $(\mathrm{AA}$ vs $\mathrm{AT}+\mathrm{TT}$ : $\left.\mathrm{P}_{\text {Beggs }}=0.015 ; \mathrm{P}_{\text {Eggers }}=0.002\right)$, and by other genotyping methods under homozygote model (AA vs TT: $\mathrm{P}_{\text {Beggs }}=0.488 ; \mathrm{P}_{\text {Eggers }}=0.016$ ). Thus, we utilized the trim-and-fill method developed by Duval and Tweedie to adjust these biases. However, after trimming we have yield similar results, indicating that the results were statistically reliable.

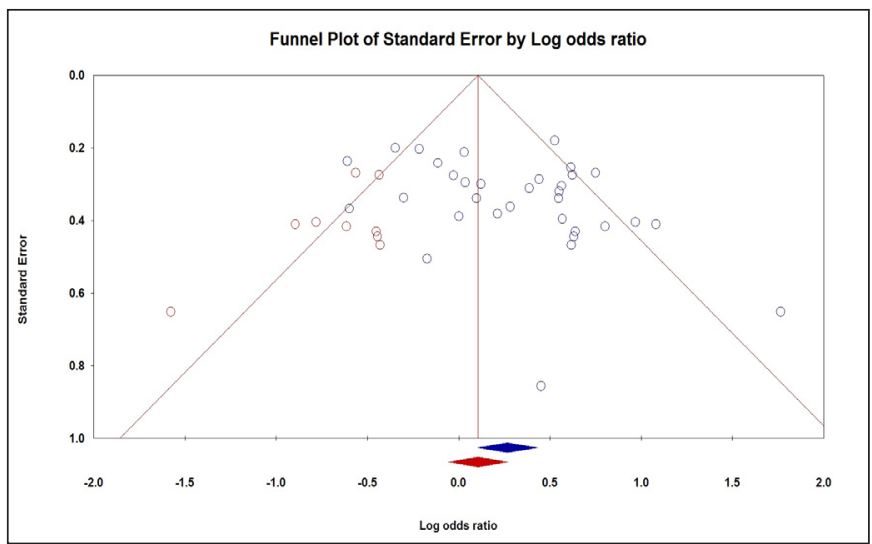

FIGURE 3. Begg's funnel of the Egger's test for publication bias test before (Blue) and after (Red) Trim-and-Fill method for association of IL-8 $-251 \mathrm{~T}>\mathrm{A}$ polymorphism with gastric cancer under homozygote model (AA vs TT). Each point represents a separate study for the indicated association.

\section{DISCUSSION}

In this meta-analysis, we included 33 case-control studies with 6,192 cases and 9,567 controls about association between IL-8 $-251 \mathrm{~T}>\mathrm{A}$ polymorphism and gastric cancer. Our pooled data showed that there was a significant association between IL-8 -251T>A polymorphism and gastric cancer under all five genetic models. Our subgroup meta-analysis also demonstrates that the IL-8 -251T>A polymorphism was associated with gastric cancer in Asians and mixed populations. Moreover, the IL-8 $-251 \mathrm{~T}>\mathrm{A}$ polymorphism is associated with gastric cancer in Chinese, Korean and Brazilian. Although, meta-analyses have revealed evidence that IL-8 -251T $>$ A polymorphism is associated with significantly increased risk of gastric cancer in overall analyses, none reported the association between IL-8 -251T >A polymorphism in mixed population and by country.

In 2015, Zhang et al., in meta-analysis of 26 studies with 5286 cases and 8000 controls have evaluated association between IL-8 -251T>A polymorphism and gastric cancer ${ }^{(40)}$. Their results showed that IL-8 -251T $>$ A polymorphism was significantly associated with increased risk of gastric cancer. Similarly, they have found that this polymorphism was associated with gastric cancer in Asians, but not in Caucasians. However, inconsistence with our pooled data, their results failed to show an association in PCR-RFLP studies. In 2018, Wang et al., in a meta-analysis of 31 studies with 5848 cases and 8926 controls have evaluated of IL-8 -251T >A polymorphism with gastric cancer ${ }^{(41)}$. Their results showed a significant association between IL-8 -251T > A polymorphism and gastric cancer risk in overall and in Asians. However, their pooled data were based on crude pooled ORs, not adjusted OR values such as genotyping methods and source of controls, which might be caused to inaccurate results. Our results seem to confirm and establish the trend in the meta-analysis of association between IL-8 -251T > A polymorphism and gastric cancer risk that the data by previous meta-analyses had indicated. Moreover, the previous meta-analyses have not performed subgroup analysis by ethnicity in mixed populations and by country. Although, our subgroup analysis showed that IL-8 -251T > A polymorphism with gastric cancer, there was no significant association in Japanese. It is suggested that the lack of an increased risk of gastric cancer in Japanese might be attributing to genetic backgrounds and environmental factors of the population. In addition, other factors such as gene-gene interactions may be modulating the IL-8 -251T >A polymorphism functionality in Japanese populations.

The possibility of publication bias in a meta-analysis is always a concern, especially when the number of incorporated studies is small(42-44). Although, large number of studies included in this meta-analysis, publication bias have distorted our results. It seems the studies that found any negative results of the association between IL-8 -251T>A polymorphism with gastric cancer may not have been published. Moreover, heterogeneity and confounding factors may have affected the meta-analysis. In this meta-analysis, there was a mild to high between-study heterogeneity under all five genetic models that could affect our results. This may be attributed to variations in confounding factors such as age, gender, source of controls, tumor stage, pathological type, and genotyping $\operatorname{method}^{(45-47)}$. We were unable to take most of these confounders into consideration in our meta-analysis because the majority of studies either did not report these baseline data. However, subgroup analyses by ethnicity, country, source of controls and genotyping methods revealed that ethnicity and country (population) may contribute to the heterogeneity in this meta-analysis. 
Our meta-analysis has several strengths. First, a systematic review of the association of IL-8 $-251 \mathrm{~T}>\mathrm{A}$ polymorphism with gastric cancer risk is statistically more powerful than any previous meta-analysis. Second, the quality of eligible studies included in this meta-analysis was satisfactory and met the inclusion criterion. Although, we performed a comprehensive meta-analysis with several subgroup analyses, there were still several limitations to be taken into consideration in this meta-analysis. First, we only selected published studies electronically in some databases, so it is possible that some pertinent studies not included in these databases or unpublished studies with negative results may have been missed. Second, only small numbers of studies were included in some subgroups such as subsets of studies among Caucasians and TaqMan. Therefore, these subgroup analyses may not have enough statistical power with the small sample size and the conclusions must be interrupted by caution. Third, although the overall sample size is large, the size of study performed in Caucasians mixed populations was relatively small. Therefore, more studies in Caucasians mixed populations are required in other populations. Finally, the mechanism of gastric cancer is considered to be sophisticated, including gene-gene and gene-environment interactions. More studies with enough statistical power are needed for deeply evaluation.

In summary, this meta-analysis suggested that the IL-8 -251T $>$ A polymorphism might contribute to susceptibility and development of gastric cancer in overall population, especially in Asians and mixed populations. Moreover, there was a significant association between IL-8 -251T > A polymorphism and an increased risk of gastric cancer in Chinese, Korean and Brazilian populations. However, it is necessary to conduct large sample studies using standardized unbiased genotyping methods, homogeneous cancer patients and well-matched controls. Moreover, further studies estimating the effect of gene-gene and gene-environment interactions may eventually lead to our better, comprehensive understanding of the association between IL-8 $-251 \mathrm{~T}>\mathrm{A}$ polymorphism and gastric cancer risk.

\section{Authors' contribution}

Moghimi M and Dastgheib SA contributed to the concept and design of the study. Neamatzadeh $\mathrm{H}$ and Zare $\mathrm{M}$ contributed to data acquisition, analysis, and interpretation. Heiranizadeh $\mathrm{N}$ and Sheikhpour E contributed to writing and editing the manuscript. All authors commented on drafts of the paper and have approved the final draft of the manuscript.

\section{Orcid}

Mansour Moghimi: 0000-0002-4000-9953.

Seyed Alireza Dastgheib: 0000-0003-4781-301X.

Naeimeh Heiranizadeh: 0000-0002-7362-4039.

Mohammad Zare: 0000-0001-9154-5273.

Elnaz Sheikhpour: 0000-0002-5622-3313.

Hossein Neamatzadeh: 0000-0003-1031-9288.

Moghimi M, Dastgheib SA, Heiranizadeh N, Zare M, Sheikhpour E, Neamatzadeh H. Associação de polimorfismo IL-8 -251T>A (rs4073) com suscetibilidade ao câncer gástrico: uma revisão sistemática e meta-análise com base em 33 estudos caso controle. Arq Gastroenterol. 2020;57(1):91-9.

RESUMO - Contexto - O papel do polimorfismo -251A>T no gene anti-inflamatório citocina interleucina-8 (IL-8) no câncer gástrico foi intensamente avaliado, mas os resultados desses estudos foram inconsistentes. Objetivo - Portanto, realizamos uma meta-análise para fornecer dados abrangentes sobre a associação de IL-8 -251T>A polimorfismo com câncer gástrico. Métodos - Todos os estudos elegíveis foram identificados nos bancos de dados PubMed, Web of Science, EMBASE, Wanfang e CNKI antes de 01 de setembro de 2019. As relações de probabilidades agrupadas (ORs) com intervalos de confiança de $95 \%$ (IC) foram derivadas de um modelo de efeito fixo ou efeito aleatório. Resultados - Foram selecionados 33 estudos de controle de caso com 6.192 casos e 9.567 controles. No geral, dados agrupados mostraram que o polimorfismo IL-8 -251T >A foi significativamente associado a um risco aumentado de câncer gástrico em todos os cinco modelos genéticos, isto é, alelo (A vs T: OR=1,189; 95\% CI 1,027-1,378; $P=0,021$ ), homozigoto (AA vs TT: $\mathrm{OR}=1,307 ; 95 \% \mathrm{CI} 1,111-1,536 ; P=0,001$ ), heterozigoto (AT vs TT: OR=1,188; $95 \% \mathrm{CI} 1,061-1,330 ; P=0,003$ ), dominante (AA+AT vs TT: $\mathrm{OR}=1,337 ; 95 \%$ CI $1,115-1,602 ; P=0,002)$ e recessivo (AA vs AT+TT: OR=1,241; 95\% CI 1,045-1,474; $P=0,014$ ). A análise estratificada por etnia revelou um risco aumentado de câncer gástrico em asiáticos e populações mistas, mas não em caucasianos. Além disso, estratificado por país. Encontrou-se uma associação significativa em chineses, coreanos e brasileiros, mas não entre os japoneses. Conclusão - Esta meta-análise sugere que o polimorfismo IL-8 -251T>A está associado a um risco aumentado de câncer gástrico, especialmente por etnia (populações asiáticas e mistas) e por país (chinês, coreano e brasileiro).

DESCRITORES - Neoplasias gástricas. Interleucina-8. Polimorfismo genético. Metanálise.

\section{REFERENCES}

1. Namazi A, Forat-Yazdi M, Jafari MA, Foroughi E, Farahnak S, Nasiri R, et al. Association between Polymorphisms of ERCC5 Gene and Susceptibility to Gastric Cancer: A Systematic Review and Meta-Analysis. Asian Pac J Cancer Prev. 2017; 18:2611-7.

2. Moghmi M, Arjmandi A, Aghili K, Jafari M, Zare-Shehneh M, Rastegar S, et al. Association of interleukin-10 -592A $>$ C AND -819T $>$ C polymorphisms with gastric cancer risk: a systematic review and meta-analysis of 44 case-control studies. Arq Bras Cir Dig. 2019;32:e1415.

3. Rawla P, Barsouk A. Epidemiology of gastric cancer: global trends, risk factors and prevention. Prz Gastroenterol. 2019;14:26-38.

4. Forman D, Burley VJ. Gastric cancer: global pattern of the disease and an overview of environmental risk factors. Best Pract Res Clin Gastroenterol. 2006;20:633-49.

5. McLean MH, El-Omar EM. Genetics of gastric cancer. Nat Rev Gastroenterol Hepatol. 2014;11:664-74.
6. Ramos MFKP, Ribeiro U, Viscondi JKY, Zilberstein B, Cecconello I, Eluf-Neto J. Risk factors associated with the development of gastric cancer - case-control study. Rev. Assoc. Med. Bras. 2018;64:611-9. doi:10.1590/1806-9282.64.07.611

7. Baggiolini M, Loetscher P, Moser B. Interleukin-8 and the chemokine family. Int J Immunopharmacol. 1995;17:103-8.

8. Futosi K, Fodor S, Mócsai A. Neutrophil cell surface receptors and their intracellular signal transduction pathways. Int Immunopharmacol. 2013;17:638-50.

9. Ha H, Debnath B, Neamati N. Role of the CXCL8-CXCR $1 / 2$ axis in cancer and inflammatory diseases. Theranostics. 2017;7:1543-88.

10. Wysoczynski M, Shin DM, Kucia M, Ratajczak MZ. Selective upregulation of interleukin- 8 by human rhabdomyosarcomas in response to hypoxia: Therapeutic implications. Int J Cancer. 2010;126:371-81.

11. Wang X Bin, Li YS, Li J, Han Y, Liu ZD. Interleukin-8 -251A/T gene polymorphism and lung cancer susceptibility: A meta-analysis. J Cell Mol Med. 2015;19:1218-22. 
12. Lee KE, Khoi PN, Xia Y, Park JS, Joo YE, Kim KK, et al. Helicobacter pylori and interleukin-8 in gastric cancer. World J Gastroenterol. 2013;19:8192-202.

13. White JR, Winter JA, Robinson K. Differential inflammatory response to Helicobacter pylori infection: Etiology and clinical outcomes. J Inflamm Res. 2015;8:137-47.

14. Zhang M, Fang T, Wang K, Mei H, Lv Z, Wang F, et al. Association of polymorphisms in interleukin-8 gene with cancer risk: A meta-analysis of 22 case-control studies. OncoTargets and Therapy. 2016;9:3727-37.

15. Li Y, Bai J, He B, Wang N, Wang H, Liu D. Weak association between the interleukin-8 rs4073 polymorphism and acute pancreatitis: a cumulative meta-analysis. BMC Medical Genetics. 2019;20(1).

16. Savage SA, Abnet CC, Haque K, Mark SD, Qiao YL, Dong ZW, et al. Polymorphisms in interleukin $-2,-6$, and -10 are not associated with gastric cardia or esophageal cancer in a high-risk chinese population. Cancer Epidemiol Biomarkers Prev. 2004;13:1547-9.

17. Lee JY, Kim HY, Kim KH, Kim SM, Jang MK, Park JY, et al. Association of polymorphism of IL-10 and TNF-A genes with gastric cancer in Korea. Cancer Letters. 2005;225:207-14.

18. Li ZW, Wu Y, Sun Y, Liu LY, Tian MM, Feng GS, et al. Inflammatory cytokine gene polymorphisms increase the risk of atrophic gastritis and intestinal metaplasia. World J Gastroenterol. 2010;16:1788-94.

19. Sun F, Sun Y, Zhang D, Zhang J, Song B, Zheng H. Association of interleukin-10 gene polymorphism with cachexia in Chinese patients with gastric cancer. Annals of clinical and laboratory science. 2010;40:149-55.

20. Bo S, Dianliang Z, Hongmei Z, Xinxiang W, Yanbing Z, Xiaobo L. Association of Interleukin-8 Gene Polymorphism With Cachexia From Patients With Gastric Cancer. J Interferon Cytokine Res. 2010;30:9-14

21. Zhang L, Du C, Guo X, Yuan L, Niu W, Yu W, et al. Interleukin-8-251A/T polymorphism and Helicobacter pylori infection influence risk for the development of gastric cardiac adenocarcinoma in a high-incidence area of China. Mol Biol Rep. 2010;37:3983-9.

22. Vinagre RMDF, Corvelo TC de O, Arnaud VC, Leite ACK, Barile KA dos S, Martins LC. Determination of strains of Helicobacter pylori and of polymorphism in the interleukin- 8 gene in patients with stomach cancer. Arq Gastroenterol. 2011;48:46-51.

23. Felipe AV, Silva TD, Pimenta CA, Kassab P, Forones NM. Interleukin-8 gene polymorphism and susceptibility to gastric cancer in a Brazilian population. Biol Res. 2012;45:369-74.

24. Burada F, Angelescu C, Mitrut P, Ciurea T, Cruce M, Saftoiu A, Ioana M. Interleukin-4 receptor $-3223 \mathrm{C} \rightarrow \mathrm{T}$ polymorphism is associated with increased gastric adenocarcinoma risk. Can J Gastroenterol. 2012;26:532-6.

25. Pan XF, Wen Y, Loh M, Wen YY, Yang SJ, Zhao ZM, et al. Interleukin-4 and -8 gene polymorphisms and risk of gastric cancer in a population in Southwestern China. Asian Pac J Cancer Prev. 2014;15:2951-7.

26. Qadri Q, Rasool R, Afroze D, Naqash S, Gulzar GM, Yousuf A, et al. Study of TLR4 and IL-8 gene polymorphisms in H. pylori-induced inflammation in gastric cancer in an ethnic kashmiri population. Immunol Invest. 2014;43:324-36

27. Kumar S, Kumari N, Mittal RD, Mohindra S, Ghoshal UC. Association between pro-(IL-8) and anti-inflammatory (IL-10) cytokine variants and their serum levels and H. pylori-related gastric carcinogenesis in northern India. Meta Gene. 2015;6:9-16

28. Taguchi A, Ohmiya N, Shirai K, Mabuchi N, Itoh A, Hirooka Y, et al. Interleukin-8 promoter polymorphism increases the risk of atrophic gastritis and gastric cancer in Japan. Cancer Epidemiol Biomarkers Prev. 2005; 14(11 Pt 1):2487-93.

29. de Oliveira JG, Rossi AFT, Nizato DM, Cadamuro AC, Jorge YC, Valsechi MC, et al. Influence of functional polymorphisms in TNF- $\alpha$, IL-8, and IL-10 cytokine genes on mRNA expression levels and risk of gastric cancer. Tumor Biology. 2015;36:9159-70

30. Wang YM, Li ZX, Tang FB, Zhang Y, Zhou T, Zhang L, et al. Association of genetic polymorphisms of interleukins with gastric cancer and precancerous gastric lesions in a high-risk Chinese population. Tumor Biology. 2016;37:2233-42.
31. Ramis IB, Vianna JS, Gonçalves CV, von Groll A, Dellagostin OA, da Silva PEA. Polymorphisms of the IL-6, IL-8 and IL-10 genes and the risk of gastric pathology in patients infected with Helicobacter pylori. J Microbiol Immunol Infect. 2017;50:153-9.

32. Chang YW, Oh CH, Kim JW, Lee JW, MJ, Shim JJ, et al. Combination of Helicobacter pylori infection and the interleukin $8-251 \mathrm{~T}>$ A polymorphism, but not the mannose-binding lectin 2 codon $54 \mathrm{G}>$ A polymorphism, might be a risk factor of gastric cancer. BMC Cancer. 2017;17(1). doi:10.1186/s12885-017-3378-2

33. Shirai K, Ohmiya N, Taguchi A, Mabuchi N, Yatsuya H, Itoh A, et al. Interleukin-8 gene polymorphism associated with susceptibility to non-cardia gastric carcinoma with microsatellite instability. J Gastroenterol Hepatol. 2006;21:1129-35.

34. Kamali-Sarvestani E, Merat A, Talei A-R. Polymorphism in the genes of alpha and beta tumor necrosis factors (TNF- $\alpha$ and TNF- $\beta$ ) and gamma interferon (IFN- $\gamma$ ) among Iranian women with breast cancer. Cancer Letters. 2005;223:113-9.

35. Kamangar F, Abnet CC, Hutchinson AA, Newschaffer CJ, Helzlsouer K, Shugart YY, et al. Polymorphisms in Inflammation-related Genes and Risk of Gastric Cancer (Finland). Cancer Causes Control. 2006;17:117-25

36. Delgado-Enciso I, Martínez-Garza SG, Rojas-Martínez A, Espinoza-Gómez F, Canseco-Avila LM, Vidal-Gutiérrez O, et al. [Article in Spanish]. [The effect of MTHFR polymorphisms, pregnancy and first intercourse on cervical cancer in a population from the Northeastern Mexico]. Rev Invest Clin. 2006;58:462-9.

37. Canedo P, Castanheira-Vale AJ, Lunet N, Pereira F, Figueiredo C, Gioia-Patricola $\mathrm{L}$, et al. The interleukin-8-251*T/*A polymorphism is not associated with risk for gastric carcinoma development in a Portuguese population. European Journal of Cancer Prevention. 2008;17:28-32. doi:10.1097/CEJ.0b013e32809b4d0f

38. Szoke D, Molnar B, Solymosi N, Klausz G, Gyulai Z, Toth B, et al. T-251A polymorphism of IL-8 relating to the development of histological gastritis and G-308A polymorphism of TNF- $\alpha$ relating to the development of macroscopic erosion. Eur J Gastroenterol Hepatol. 2008;20:191-5.

39. Song B, Wang S, Zheng H, Wang X. Association of interleukin-8 with cachexia from patients with low-third gastric cancer. Comparative and Functional Genomics. 2009;2009. doi:10.1155/2009/212345

40. Zhang Y, Zeng X, Lu H, Li Y, Ji H. Association between Interleukin-8-251A/T polymorphism and gastric cancer susceptibility: a meta-analysis based on 5286 cases and 8000 controls. Int J Clin Exp Med. 2015;8:22393-402.

41. Wang X, Yang F, Xu G, Zhong S. The roles of IL-6, IL-8 and IL-10 gene polymorphisms in gastric cancer: A meta-analysis. Cytokine. 2018;111:230-6.

42. Gohari M, Dastgheib SA, Noorishadkam M, Lookzadeh MH, Mirjalili SR, Akbarian-Bafghi MJ, et al. Association of eNOS and ACE Polymorphisms with Retinopathy of Prematurity: A Systematic Review and Meta-Analysis. Fetal Pediatr Pathol. 2019:1-12

43. Aflatoonian M, Moghimi M, Akbarian-Bafghi MJ, Morovati-Sharifabad M, Jarahzadeh $\mathrm{MH}$, Neamatzadeh $\mathrm{H}$. Association of TNF- $\alpha-308 \mathrm{G}>\mathrm{A}$ polymorphism with susceptibility to celiac disease: a systematic review and meta-analysis. Arq Gastroenterol. 2019;56:88-94.

44. Farbod M, Karimi-Zarchi M, Heiranizadeh N, Seifi NS, Akbarian JBM, Jarahzadeh HM, Neamatzadeh H. Association of TNF- $\alpha-308 \mathrm{G}>$ A Polymorphism with Susceptibility to Cervical Cancer and Breast Cancer - a Systematic Review and Meta-analysis. Klinicka Onkologie. 2019;32:170-80. doi:10.14735/amko2019170.

45. Moghimi M, Sobhan MR, Jarahzadeh MH, Majid Morovati-Sharifabad; Kazem Aghili; Hossein Ahrar, et al. Association of GSTM1, GSTT1, GSTM3, and GSTP1 Genes Polymorphisms with Susceptibility to Osteosarcoma: a CaseControl Study and Meta-Analysis. Asian Pac J Cancer Prev. 2019;20:675-82. doi:10.31557/APJCP.2019.20.3.675

46. Sobhan MR, Mahdinezhad-Yazdi M, Moghimi M, Aghili K, Jafari M, Zare-Shehneh M, et al. Plasminogen Activator Inhibitor-1 4G/5G Polymorphism Contributes to Osteonecrosis of the Femoral Head Susceptibility: Evidence from a Systematic Review and Meta-analysis. Arch Bone Jt Surg. 2018;6:468-77.

47. Jafari-Nedooshan J, Moghimi M, Zare M, Heiranizadeh N, Morovati-Sharifabad M, Akbarian-Bafghi MJ, et al. Association of Promoter Region Polymorphisms of IL-10 Gene with Susceptibility to Lung Cancer: Systematic Review and Meta-Analysis. Asian Pac J Cancer Prev. 2019;20:1951-7. 